\title{
In vivo imaging of cellular structures in Caenorhabditis elegans by combined TPEF, SHG and THG microscopy
}

\author{
E. J. GUALDA*, G. FILIPPIDIS ${ }^{*}$, G. VOGLIS $\dagger$, M. MARI* $\ddagger$, \\ C. FOTAKIS ${ }^{*} \ddagger \&$ N. TAVERNARAKIS $†$ \\ *Institute of Electronic Structure and Laser, Foundation of Research and Technology-Hellas, \\ P.O. Box 1527, 71110 Heraklion, Crete, Greece \\ $\dagger$ Institute of Molecular Biology and Biotechnology, Foundation of Research and Technology, 71110, \\ Heraklion, Crete, Greece \\ $\ddagger$ Department of Physics, University of Crete
}

Key words. Caenorhabditis elegans, imaging, microscopy, SHG, THG, TPEF.

\begin{abstract}
Summary
In this study, we use combined two-photon excitation fluorescence (TPEF), second-harmonic generation (SHG) and third-harmonic generation (THG) measurements to image cellular structures of the nematode Caenorhabditis elegans, in vivo. To our knowledge, this is the first time that a THG modality is employed to image live $C$. elegans specimens. Femtosecond laser pulses $(1028 \mathrm{~nm})$ were utilized for excitation. Detailed and specific structural and anatomical features can be visualized, by recording THG signals. Thus, the combination of three image-contrast modes (TPEF-SHG-THG) in a single instrument has the potential to provide unique and complementary information about the structure and function of tissues and individual cells of live biological specimens.
\end{abstract}

\section{Introduction}

In the last few years, non-linear optical measurements used in conjunction with microscopy observations have created new opportunities of research and technological achievements in the field of biology. Owing to their inherent advantages in comparison with conventional microscopy, non-linear microscopy techniques comprise a unique and extremely powerful tool for the extraction of valuable information from biological samples. Two-photon excitation fluorescence (TPEF), second-harmonic generation (SHG) and thirdharmonic generation (THG) are well-established techniques that have been recently used as tools for imaging and mapping of sub-cellular biological structures and processes, in vivo

Correspondence to: G. Filippidis. Tel: +30 2810391320; fax: +30 2810 391318; e-mail: filip@iesl.forth.gr
(Denk et al., 1990; Xu et al., 1996; Muller et al., 1998; Squier et al., 1998; Moreaux et al., 2000; Chu et al., 2002; Yeh et al., 2002; Mohler et al., 2003; Debarre et al., 2005). They exhibit intrinsic three-dimensionality and the ability to section deep within biological tissues, due to their non-linear nature. They have significant efficiency only at extremely high incident light intensities, and therefore arise only from a well-defined volume around the focal centre of the incident light beam. In TPEF, SHG and THG, the wavelength of the fundamental incident light lies in the infrared (IR) spectrum region, thus suffering less scattering and absorption inside biological samples and exhibiting larger penetration depths. SHG, THG and TPEF microscopy methodologies do not provide higher resolution compared to confocal one-photon microscopy. However, as far as the axial direction is concerned, excitation of the biological specimen in non-linear microscopy is confined in a small region around the focal plane, due to the quadratic dependence of SHG and TPEF intensities and the cubic dependence of THG intensity upon the excitation photon flux. 'Out of focal plane' photobleaching and phototoxicity are thus dramatically reduced, diminishing damage and permitting increased survival of biological specimens during in vivo experiments. Furthermore, optical higher harmonic generation, including SHG and THG, does not deposit energy to the specimen due to its energy-conservation characteristics, providing minimum sample disturbance, which is desirable for biological studies.

Despite similarities, SHG, THG and TPEF are based on fundamentally different phenomena (Bloembergen, 1965). The first two rely on non-linear scattering whereas the third relies on non-linear absorption followed by fluorescence emission. Therefore, the first two are coherent processes, whereas the third is not. TPEF microscopy is mainly used to 
visualize endogenous or exogenous fluorophores in biology (Xu et al., 1996) with good lateral and axial resolution. SHG is a useful technique for probing structures and functions of membranes and the membrane potential induced alignment of dipolar molecules (Khatchatouriants et al., 2000; Campagnola et al., 2001; Zoumi et al., 2002; Millard et al., 2003; Moreaux et al., 2003; Pons et al., 2003; Campagnola \& Loew, 2003; Zipfel et al., 2003; Dombeck et al., 2004). In principle, TPEF provides information about the concentration of chromophores within biological specimens, whereas SHG provides information about the global orientation of the biological structures. THG is generated near the interface between two media (Squier \& Muller, 2001). Thus, THG can be used to image the different organs of a transparent biological sample with a three-dimensional (3D) microscopy capability (Millard et al., 1999; Yelin \& Silberberg, 1999; Yelin et al., 2002; Chu et al., 2003; Sun et al., 2004; Tsai et al., 2006; Supatto et al., 2005; Clay et al., 2006). A specific advantage of THG is that, although SHG requires a medium without inversion symmetry, third-order processes such as THG are allowed in any medium. As reported previously (Chu et al., 2001; Yelin et al., 2002; Chu et al., 2003; Sun et al., 2004; Supatto et al., 2005; Tsai et al., 2006), a non-linear microscope can be easily adapted for measuring THG signals.

Owing to their similarities and differences, two-photon excitation fluorescence (TPEF), second- and third-harmonic generation (SHG and THG) imaging microscopy techniques have the potential to provide complementary information about functions and processes of several biological systems in vivo. The combination of these three image contrast modalities in a single instrument can be very advantageous since new insights into embryonic morphological changes and complex developmental processes are expected by exploiting all these non-linear techniques simultaneously.

Different femtosecond lasers have been used as excitation source for THG microscopy measurements. Systems emitting above $1200 \mathrm{~nm}$, as synchronously pumped OPO emitting at $1500 \mathrm{~nm}$ (Yelin \& Silberberg, 1999), femtosecond fibre laser at $1560 \mathrm{~nm}$ (Millard et al., 1999) and Cr:Forsterite emitting at $1230 \mathrm{~nm}$ (Chu et al., 2001, 2003; Sun et al., 2004; Tsai et al., 2006), have been employed because of the advantage that their third-harmonic signals are in the visible range. However, absorption in water increases by employing these wavelengths as excitation sources leading to unwanted heating of the biological specimens (especially by using wavelengths above $1500 \mathrm{~nm}$ ). In addition, the lateral and axial resolutions degrade with the increasing wavelength. On the other hand, THG microscopy measurements have been performed by using Ti:sapphire lasers $(810 \mathrm{~nm})$ (Yelin et al., 2002). The main limitation of a Ti:sapphire laser as an excitation source for THG measurements is the wavelength of the third harmonic, which is located in the ultraviolet (UV) near $270 \mathrm{~nm}$. Therefore, condenser optics and deflecting mirrors with special coatings are needed for the collection of the THG signal. Moreover, the
THG signal is completely absorbed by standard microscope slides or even from thin cover slips, imposing an inverted configuration of the microscope (Yelin et al., 2002).

THG measurements have also been performed by employing femtosecond lasers emitting at around $1 \mu \mathrm{m}$ (Clay et al., 2006). In our study, we used a femtosecond laser oscillator emitting at $1028 \mathrm{~nm}$ as an excitation source. To our knowledge, this is the first time that this wavelength is employed to produce THG signals from biological samples. The absorption coefficient of water for this wavelength is $0.1 \mathrm{~cm}^{-1}$, avoiding unwanted thermal heating of the sample (Curcio \& Petty, 1951). The emitted THG signal lies in the near-ultraviolet (UV) part of the spectrum near $343 \mathrm{~nm}$, thus common condenser lenses (Carl Zeiss Plan-Apochromat 100X/1.4 oil, Carl, Zeiss, Oberkochen, Germany) can be used for the detection of the signal. The induced losses of the emitted THG signals, due to the thin cover slips which are used to mount the biological samples, are constrained. Moreover, by using the $1028 \mathrm{~nm}$ as excitation source, photodamage effects onto the biological specimens are reduced (owing to the lower power per photon) compared with the typical wavelength of around the $800 \mathrm{~nm}$.

Here, we present detailed imaging and mapping of the nematode Caenorhabditis elegans (C. elegans) using nonlinear microscopy (TPEF-SHG-THG). Wild-type worms and transgenic animals, which express Green Fluorescence Protein (GFP) in the pharyngeal muscle cells, have been imaged. In addition, mutants that express GFP in the cytoplasm of the six mechanoreceptor neurons have been investigated. Several research groups have performed detailed imaging of the C. elegans body, by recording non-linear phenomena (TPEF and SHG) at the microscopic level (Lewis et al., 1999; Khatchatouriants et al., 2000; Campagnola et al., 2002; Campagnola \& Loew, 2003; Mohler et al., 2003; Nikolenko et al., 2003; Filippidis et al., 2005a, 2005b), obtaining valuable information concerning the biology of the nematode. Thisis the first time that THG modality is employed to image C. elegans samples. By detecting THG signals from the worm, specific and detailed anatomical information can be retrieved. The combination of three non-linear image-contrast modalities (TPEF, SHG, THG) in a single microscope can provide unique and complementary information allowing a 3Dreconstruction of animal body features.

\section{Experimental apparatus}

The experimental set-up is outlined in Fig. 1. The laser used is an Amplitude Systems t-pulse laser operating at $1028 \mathrm{~nm}$. This source is a compact diode-pumped femtosecond laser oscillator delivering a train of high peak power, short duration pulses. The average power of the laser is $1 \mathrm{~W}$, with pulse duration of less than 200 femtoseconds and a repetition rate of $50 \mathrm{MHz}$. The beam is directed to a modified optical microscope (Nikon Eclipse ME600D, Nikon Corp., Japan) using suitable dichroic mirrors, and is focused tightly onto the sample by an objective 


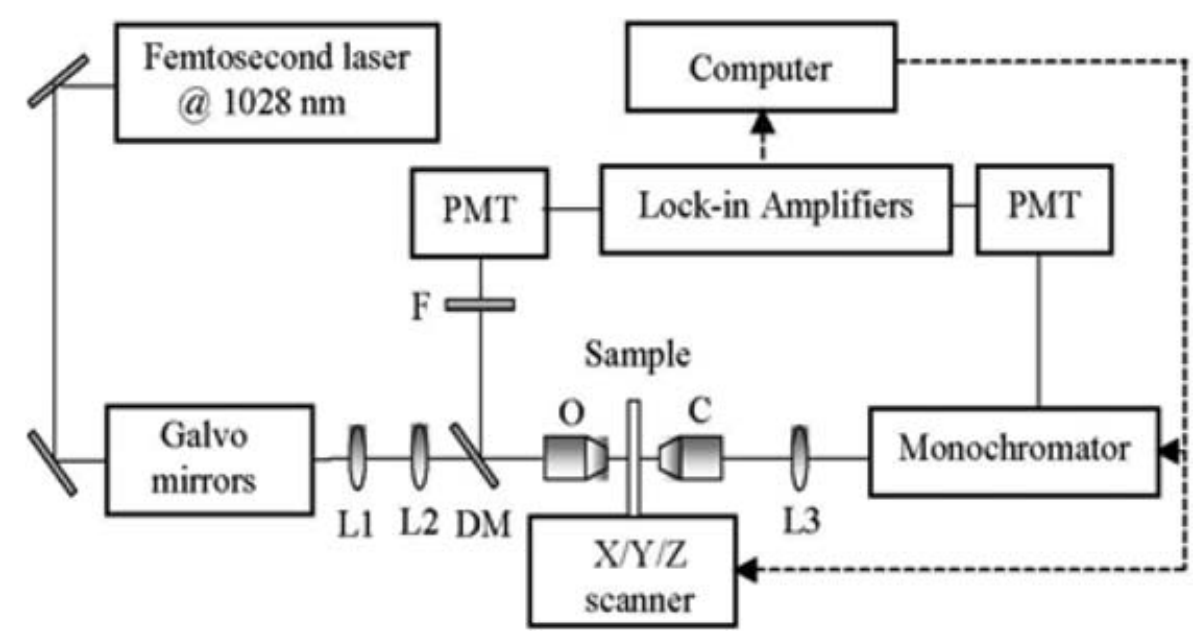

Fig. 1. Schematic representation of the experimental set-up. Two signals are collected simultaneously, TPEF in the backward and THG or SHG in the forward direction, by tuning the monochromator. The system is completely automated and controlled with a LabView interface. PMT: photomultiplier tube, DM: Dichroic mirror, O: objective, C: condenser, F: filter, L1/L2: telescope lenses and L3: lens

lens with high numerical aperture (Nikon 50X N.A. 0.8, Nikon Corp., Japan). To ensure that the back aperture of the objective is fulfilled, a telescope system has been used. The average laser power on the specimen is $30 \mathrm{~mW}$ (0.6 nJper pulse). No damage in the sample is observed at this power.

A CCD camera (PixeLINK PL A662, PixeLink, Ottawa, Canada) is used for observation. Biological samples are placed between two round glass slides (Marienfeld $0.06 \mathrm{~mm}-$ $0.08 \mathrm{~mm}$, Paul Marienfeld GmbH \& Co., KG, Germany) that fit into a motorized xyz translation stage (Standa 8MT167-100, Standa, Lithuania) by employing a special holder. The minimum step of the stages in each direction is $1 \mu \mathrm{m}$. The combined movement of the three stages is computer controlled by specially designed software (National Instruments, Labview 7.1, National Instruments, TX, USA), allowing the implementation of a complicated scanning scheme. The average accumulation time in every step is 30 ms. Alternative, by performing the scanning procedure with the use of a pair of computer-controlled galvanometric mirrors (Cambridge Tech. 6210H, Cambridge Tech Inc., MA, USA), high-resolution images can be obtained.

TPEF signals are collected in the backward direction using a photomultiplier tube (PMT Hamamatsu R4220, Hamamatsu, Japan) connected to a lock-in amplifier (SR810 Stanford Research Systems, Stanford Research Systems Inc., CA, USA). The use of a lock-in amplifier provides a very good noise rejection, and measurements can be performed in less stringent lighting conditions. The photomultiplier tube is attached at the position of the microscope eye-piece. A short pass filter (SPF $650 \mathrm{~nm} \mathrm{CVI}$ ) is placed at the photomultiplier input to cut-off the reflected laser light. Furthermore, a long pass filter (LP $520 \mathrm{~nm} \mathrm{CVI}$ ) is used for the elimination of reflected SHG (Yeh et al., 2002; Zoumi et al., 2002; Williams et al., 2005) and THG signals. For thin samples, most of SHG and THG signals propagate with the laser and are collected and collimated by employing a common condenser lens (Carl Zeiss PlanApochromat 100X N.A. 1.4 oil immersion). A dichroic mirror (Enhanced Aluminium Mirror, Thorlabs, NJ, USA) is used to reflect the transmitted beam. The signals are focused by means of a lens (UV Fused Silica Plano-Convex, Thorlabs) into the slit $(100 \mu \mathrm{m})$ of a monochromator. The focal length of the lens is $3 \mathrm{~cm}$. The monochromator (Digikrom CM110 CVI, Digikrom, NM, USA) consists of two gratings (1200 grooves/mm each). The first one is suitable for visible light (maximum efficiency $80 \%$ at $500 \mathrm{~nm}$ ) and the second is appropriate for ultraviolet light (maximum efficiency $72 \%$ at $300 \mathrm{~nm}$ ). The resolution of the monochromator is $1 \mathrm{~nm}$. For the detection of signals, a photomultiplier tube (PMT Hamamatsu R636-10) connected to another lock-in amplifier is used. With this configuration, it is possible to record SHG and THG signals in distinct sets of measurements by tuning the monochromator in different spectral regions. By detecting SHG or THG images in the forward direction and TPEF images in the backward direction, our experimental apparatus allows the collection of two nonlinear optical signals simultaneously.

\section{Biological samples}

We used the nematode $C$. elegans to exploit the capacity of combined non-linear optical imaging to provide specific structural and functional information about a biological system.

C. elegans is a small $(1 \mathrm{~mm})$ free-living hermaphroditic, nematode worm that completes a life-cycle in 2.5 days at $25^{\circ} \mathrm{C}$. The simple body plan and transparent nature of both the egg and the cuticle have facilitated the exceptionally detailed developmental and anatomical characterization of this animal. The complete sequence of cell divisions and the normal pattern of programmed cell death events that occur as the fertilized egg develops into the 959-celled 
adult are known (Sulston et al., 1983). C. elegans is the first metazoan for which the genome was sequenced to completion (The C. elegans Sequencing Consortium, 1998). An important advantage of this experimental model organism is that construction of transgenic animals is rapid. DNA injected into the hermaphrodite gonad concatamerizes and is packaged into embryos in the form of an extra chromosomal array. Hundreds of progeny can be obtained within a few days of the injection (Mello et al., 1991). The anatomical characterization and understanding of neuronal connectivity in C. elegans is unparalleled in the metazoan world. Serial section electron microscopy has identified the pattern of synaptic connections made by each of the 302 neurons of the animal (including 5000 chemical synapses, 600 gap junctions and 2000 neuromuscular junctions), so that the full 'wiring diagram' of the animal is known (White et al., 1996). Overall, the broad range of genetic and molecular techniques applicable in C. elegans allows a unique line of investigation into fundamental problems in biology.

We followed standard procedures for C. elegans strain maintenance, crosses and other genetic manipulations (Brenner, 1974). Nematode rearing temperature was kept at $20^{\circ} \mathrm{C}$. Before each experiment, young adult animals were anaesthetized by immersing to $0.5 \mathrm{M}$ of sodium azide $\left(\mathrm{NaN}_{3}\right)$, and subsequently mounted on glass slides.

\section{Results}

To demonstrate the performance of the t-pulse laser operating at $1028 \mathrm{~nm}$ as a photon source for THG measurements with biological samples at the microscopic level, different body parts of wild type C. elegans were imaged. As mentioned previously, the THG signal is generated at the interface between different media that break the axial symmetry of focus. That allows recording of THG signals arising from the worm body surface and from boundaries between organs, tissues and cells. We find that specific organs generate strong signals that reveal novel structural information about the anatomy of the nematode.

Figure 2(a) depicts the THG image of the pharynx region of a wild-type animal. Figure 2(b) shows the THG image from the posterior end and Fig. 2(c) shows the uterus region of the worm. A light-transmission picture of the scanned region is included for reference. The dimensions of the scanned area were $61 \times$ $21 \mu^{2}, 51 \times 25 \mu^{2}$ and $121 \times 55 \mu^{2}$, respectively. Signals were recorded in the forward direction, as described previously. The monochromator was tuned at $343 \mathrm{~nm}$. Thus, detection was performed in a spectral region where the only detectable signal is contributed by THG. Each scan was performed in a specific $z$ position where the THG signal that arises from the sample was maximal.

From Fig. 2, the shape and contour of the worm as well as the internal tissue and organ arrangement, can be observed with satisfactory resolution. It isfeasible the collection of strong THG signals originate from the body surface, the pseudocoelomic cavity and the boundaries between internal organs of the worm, due to abrupt changes of the refractive index values (Fig. 2(a)). This indicates constitution differences between different tissues. The collected THG signals from the pharynx are minimal. Similar characteristics can be seen in Fig. 2(b).

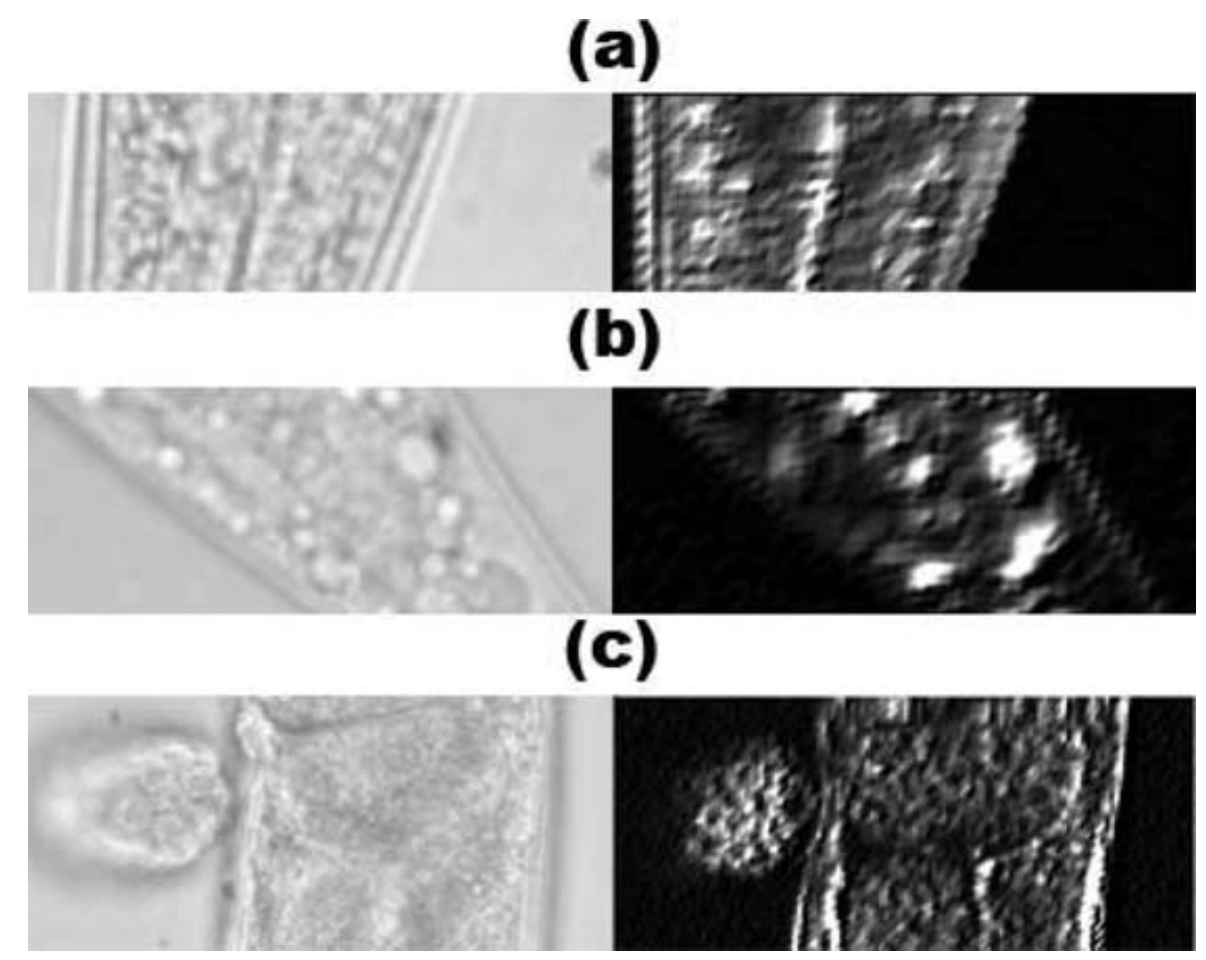

Fig. 2. THG images from (a) the pharynx region, (b) the tail and (c) the mid-body section of wild type C. elegans. 
The outline of the worm tail is clearly visible. High THG signals were generated by discontinuities in the region of the tail. These discontinuities are likely due to lipid depositions (Debarre et al., 2006) or formations of discontinuous refractive index in the intestine and pseudocoelomic cavity. The central part of the worm is shown in Fig. 2(c). Three eggs are also shown, two inside the uterus and another in the vicinity of the animal. The contours of individual cells inside the external egg are detectable through THG measurements. No THG signal is recorded from the left part of the external egg, which appears slightly defocused in the light-transmission picture, demonstrating the sectioning capabilities of THG measurements. We note that the signals are obtained without the use of labelling molecules.

As shown previously, THG is a general-purpose technique that provides structural information about the sample, but is not capable to provide information on specific fluorescent molecules. To improve and expand the imaging capacity of our microscope, we sought to combine THG measurements with complementary non-linear techniques. Because of their specific nature, the three non-linear signals described earlier (TPEF-SHG-THG) are generated by different structures and molecules in the animals. By recording simultaneously these three non-linear modalities, cumulative information about the biological sample is obtained. The use of an excitation wavelength of $1028 \mathrm{~nm}$ allows us to record all three signals with a single laser, simplifying the layout of the set-up. In addition, with this configuration, we ensure that produced signals correspond to the same optical section.

Figure 3 depicts the non-linear imaging (TPEF-SHG-THG) of the C. elegans anterior part. In these transgenic animals, GFP is expressed in the cytoplasm of the pharyngeal muscle cells. The dimensions of the scanned region were $51 \times 21 \mu \mathrm{m}^{2}$. Scanning was performed at a specific $z$ position where the collected TPEF signal derived from the pharynx became maximum. Figure 3(a) shows a TPEF image, obtained by backward detection, whereas Figs 3(b) and (c) show the SHG and THG measurements, respectively, detected in the forward direction. SHG and THG measurements were performed in different sets by tuning the monochromator at $514 \mathrm{~nm}$ and $343 \mathrm{~nm}$, respectively. There are main peaks at $514 \mathrm{~nm}$ (SHG) and $343 \mathrm{~nm}$ (THG) which abruptly reduce, as the monochromator setting was changed 3-4 $\mathrm{nm}$ around these wavelengths. These observations are in perfect agreement with the spectral distributions, which present the non-linear phenomena of second- and third-harmonic generation. Moreover, it is worth mentioning that by placing a 514-nm interference filter (CVI F03-514.5) or a 340-nm band pass filter (UQG Hoya U 340 UV transmitting Vis and IR absorbing, Hoya, CA, USA) in the forward detection scheme, the recorded SHG or THG images from the nematode, respectively, are similar with the ones obtained without the filters. Consequently, the contribution of the higher diffraction orders of the fundamental beam, due to the use of gratings, can be safely excluded from the detected
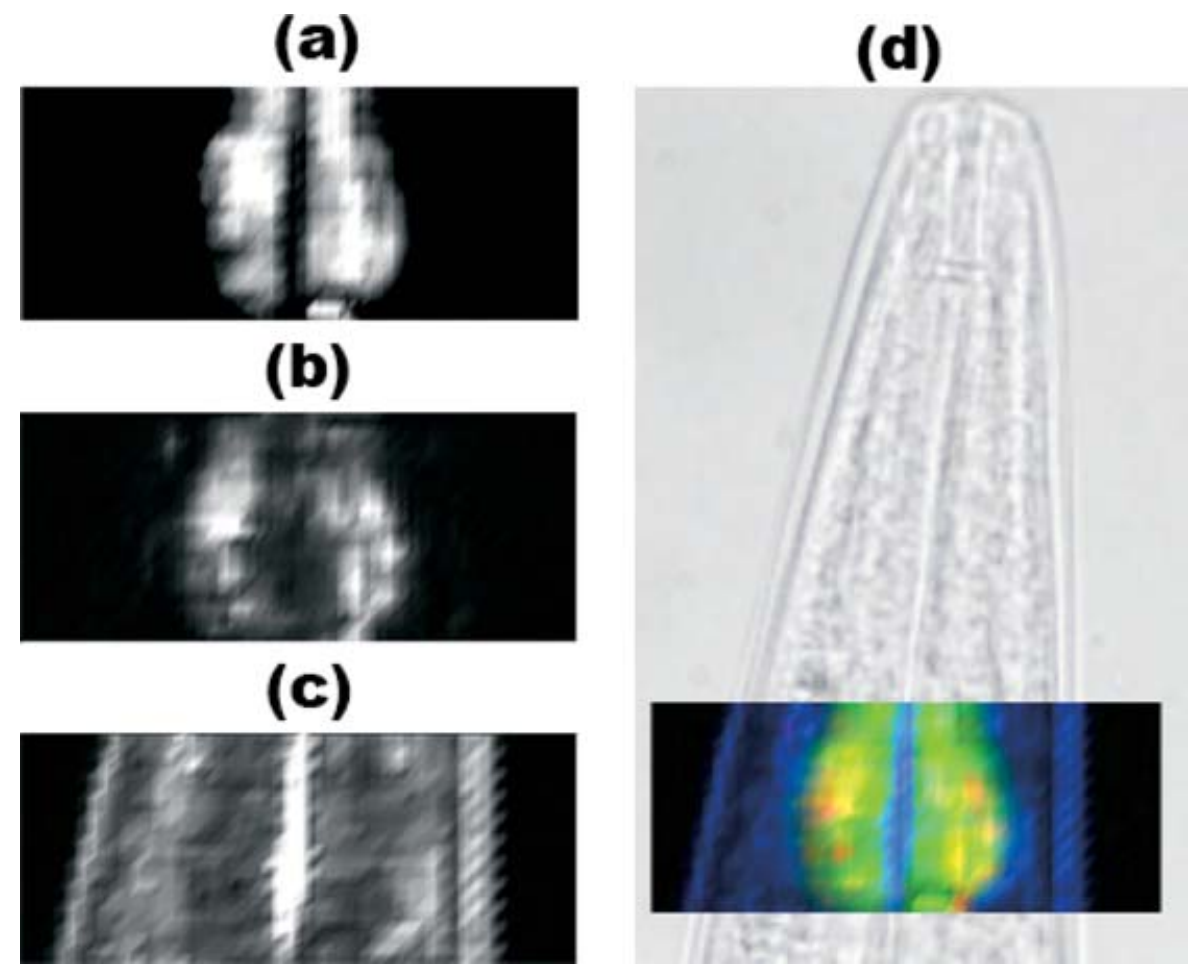

Fig. 3. Non-linear signals generated in the pharynx region of a worm expressing GFP in the pharyngeal muscles: (a) TPEF, (b) SHG, (c) THG and (d) multi-modal image obtained by the combination of the previous three: TPEF (green), SHG (red) and THG (blue). 

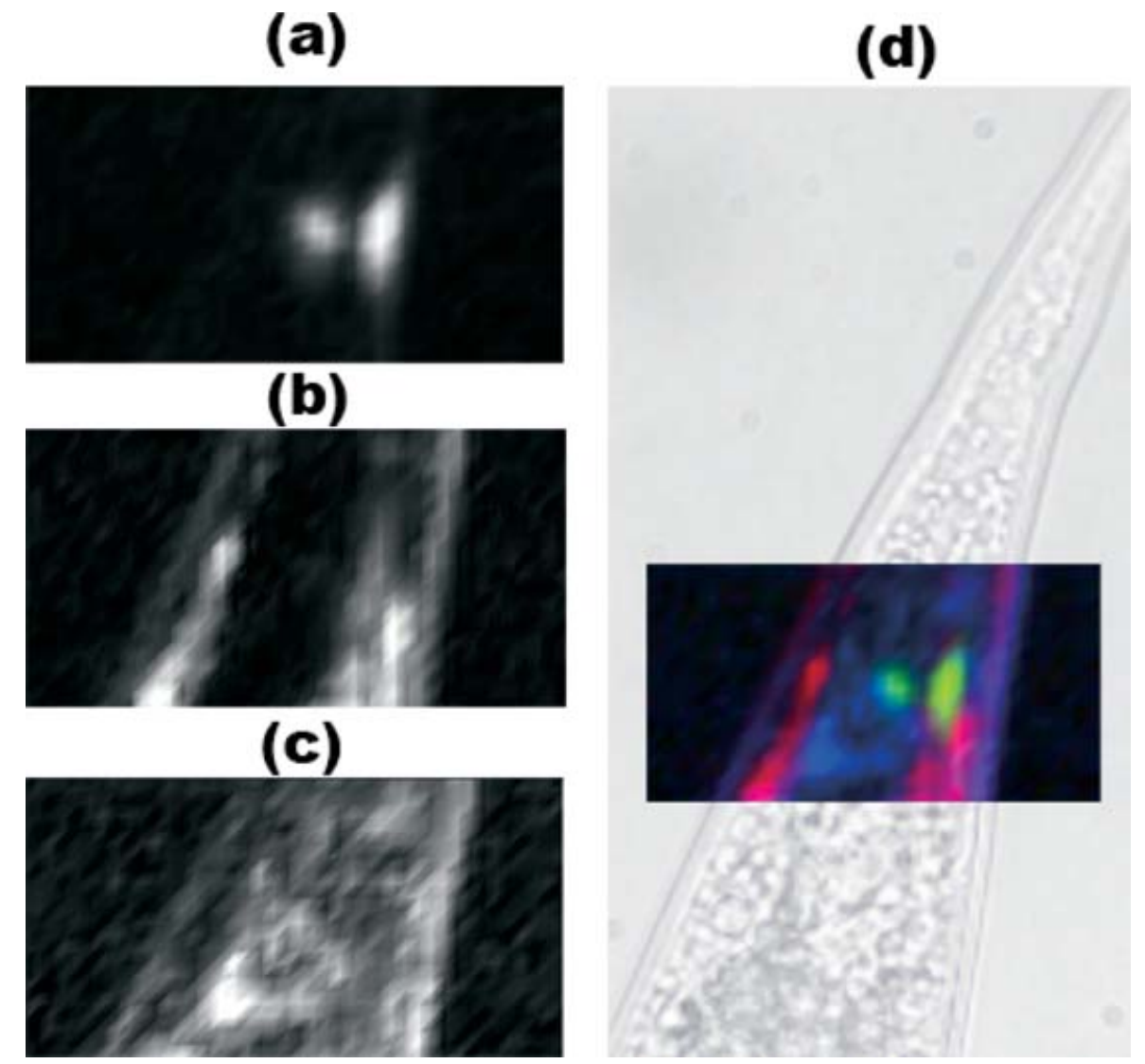

Fig. 4. Non-linear signals generated at the tail of an animal expressing GFP in the mechanoreceptor neurons: (a) TPEF, (b) SHG, (c) THG and (d) multi-modal image obtained by the combination of the previous three: TPEF (green), SHG (red) and THG (blue).

signals. The combination of the three contributions is shown in Fig. 3(d) (TPEF in green, SHG in red and THG in blue). By TPEF imaging of the GFP molecules in pharyngeal muscles, we are able to visualize the inner part of the pharynx. The endogenous structures of well-ordered protein assemblies in the pharyngeal muscles, such as actomyosin complexes, are the main contributors to recorded SHG signals (Campagnola et al., 2002; Campagnola \& Loew, 2003; Mohler et al., 2003; Filippidis et al. 2005a, 2005b; Plotnikov et al. 2006). GFP is expressed under the control of the myo- 2 promoter in animals used for this experiment. The myo-2 promoter is specific to the pharynx and GFP expression is limited to the cytoplasm of pharyngeal muscle cells. GFP molecules, due to their random orientation in the pharynx region do not contribute to the SHG signal. The contour and shape of the worm can be observed through THG measurements. Furthermore, high THG intensity signals were collected from the linings of the animal pseudocoelomic cavity. The three image modalities provide complementary information about the biological sample, as seen in Fig. 3. This is due to the fact that individual, induced signals come from different components. Diffuse GFP molecules are the main source of the detected TPEF signals. The endogenous oriented structural proteins are responsible for the observed SHG signals. Unique structural and functional information can be obtained by recording THG signals, given the sensitivity of these signals to changes of the refractive index of the medium.

Figure 4 depicts non-linear imaging (TPEF-SHG-THG) of the posterior part of a sample. In these experiments, GFP is expressed under the mec- 4 touch-cell-specific promoter and is localized in the cytoplasm of the six mechanoreceptor neurons of the animal. Two of the six mechanoreceptor neurons are located in the posterior part of the worm, usually in different $z$ positions. These two touch receptor neurons (PLML-PLMR) do not overlap with the gut. Their somata are situated close to the tip of the tail, away from the gut auto-fluorescence. The dimensions of the scanned region were $41 \times 21 \mu \mathrm{m}^{2}$. Scanning was performed at a specific $z$ position, where the TPEF signal emanating from one neuron was maximum. The recorded TPEF signal arises from cytoplasmic GFP molecules, which are expressed in the six neurons. It is clear that the precise localization of the two touch receptor neurons, which are positioned near the tail, is feasible by processing of the obtained images (Fig. 4(a)). The SHG image enabled us to detect muscles close to the tail of the worm. Endogenous structural proteins (actomyosin complexes) 
are the main contributors to the recorded SHG signals (Filippidis et al., 2005a; Plotnikov et al., 2006). GFP molecules in the neuronal cell are symmetrically distributed. Therefore, their contribution to the observed SHG signal is expected to be minimal. This is in agreement with experimental data, since it was not possible to detect the neuronal cells, as depicted in Fig. 4(b). In Fig. 4(c), the morphology and the contour of the worm can be observed through THG measurements. Rather, discontinuities (such as sub-cellular organelles, inclusions, cavities, lipid depositions, etc.) in the C. elegans posterior body segment are the source of high-intensity THG signals. Figure 4(d) shows the superposition of the three non-linear signals. It is again clear that the three types of non-linear imaging (TPEF, SHG and THG) provide complementary information.

We point out that, by collecting THG images, detailed information related to the structure, the contour and the discontinuities of the animal tail are obtained (shown in Figs 3 and 4). This information provides a frame of reference, which facilitates the precise localization of specific stained markers by fluorescence measurements and allowing structural orientation studies through SHG experiments.

Of special interest is the capacity for 3D reconstruction of a live organism that should facilitate the detailed description of anatomical features of both wild type and genetically modify animals. Such reconstructions may contribute to the understanding of the molecular mechanisms pertinent to specific genetic alterations. Since THG mainly provides information about boundaries between different media, it is feasible to reconstruct the animal outer surface and extract structural information from several inner organs by performing scan at different $z$ positions. To this end, we utilized the Matlab software package (MathWorks, Ink, MA, USA) to design appropriate routines.

Figure 5 shows the 3D reconstruction of non-linear (THG and TPEF) images obtained from the anterior part of C. elegans. The strain used in this line of experiments expressed GFP in the cytoplasm of the pharyngeal muscle cells. The dimensions of the scanning region were $71 \times 21 \mu^{2}$. Five optical sections, $5 \mu \mathrm{m}$ apart, were collected. Figures $5(\mathrm{a})$ and (b) present the z-projection of the TPEF and THG images, respectively. The two different non-linear images (TPEF and THG) were recorded simultaneously in each optical section. Figure 5(c) is the superposition of the two 3D reconstructed images. Because GFP molecules are localized inside the pharynx of the nematode, the outline of the pharynx can be recognized (red surface) from the reconstruction of the TPEF images. Conversely, the contour of the worm body is becomes obvious (grey surface) by the reconstruction of THG images. It is important to point out, the difference in the origin of TPEF and THG signals. Whereas THG provides information about the morphological features of the worm and internal organs, TPEF indicates the position of the fluorescent protein.

The 3D reconstruction of THG and TPEF images, recorded from the posterior part of the nematode is shown in Fig. 6 .
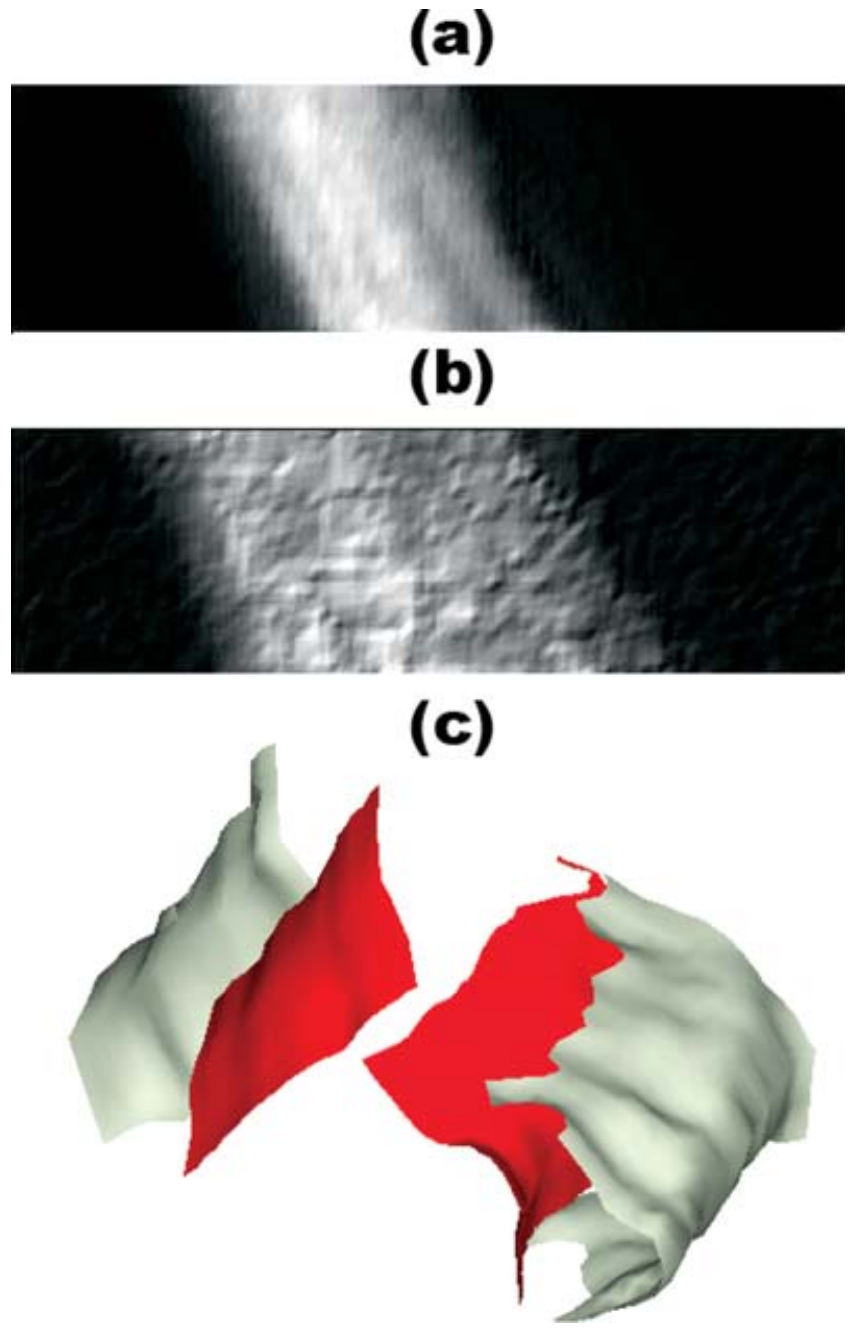

Fig. 5. Z-projection of non-linear signals generated in the pharynx region of a worm expressing GFP in pharyngeal muscles. The projection comprises five slices $5 \mu \mathrm{m}$ apart: (a) TPEF and (b) THG. The 3D reconstruction is shown in (c).

We used strains that express GFP in the cytoplasm of the six mechanoreceptor neurons of C. elegans. The dimensions of the scanned region were $41 \times 21 \mu \mathrm{m}^{2}$. Eight optical sections, $5 \mu \mathrm{m}$ apart, were obtained. Figure 6(a) shows the z-projection of TPEF images, Fig. 6(b) depicts the corresponding THG images and Fig. 6(c) is the superposition of the two (TPEF and THG) 3D reconstructed images. The precise 3D localization of the two touch receptor neurons which are positioned near the tail of the animal is easily derived (red surface) from the reconstruction of TPEF images (due to fluorescence of the cytoplasmic GFP molecules). Moreover, detailed morphological information concerning the shape (grey surface) and the inner structure of the animal tail (blue surface) can be obtained from the reconstruction of THG images.

The resolution of our non-linear system is limited to $1 \mu \mathrm{m}$ due to the use of the xyz motorized stage (Standa). This 


\section{(a)}

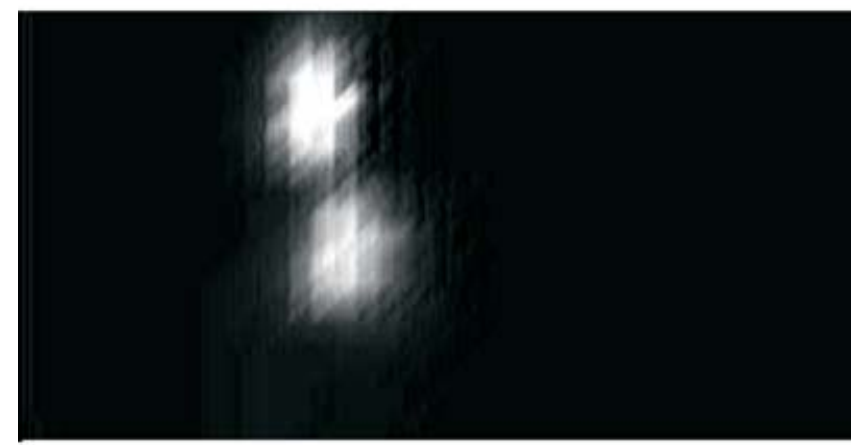

(b)
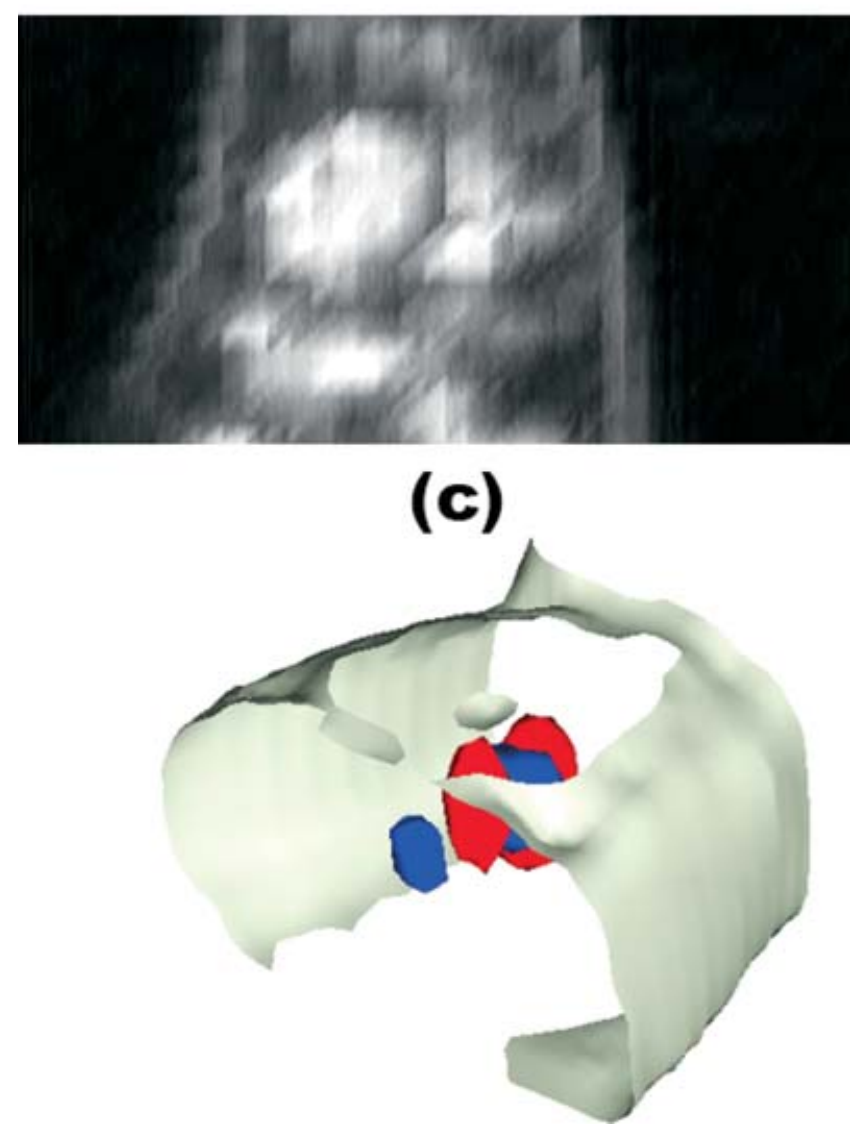

Fig. 6. Z-projection of the non-linear signals generated in the tail of a worm expressing GFP in the mechanoreceptor neurons. The projection corresponds to eight slices every $5 \mu \mathrm{m}$ : (a) TPEF and (b) THG. The 3D reconstruction is shown in (c).

choice represents an inexpensive and reliable solution for the realization of the scanning procedure. To verify, in a more accurate way, which anatomical features give rise to the THG signals and to improve the quality and resolution of the collected images, we performed a number of experiments by replacing the motorized stage with a galvano-mirror scanning (a)
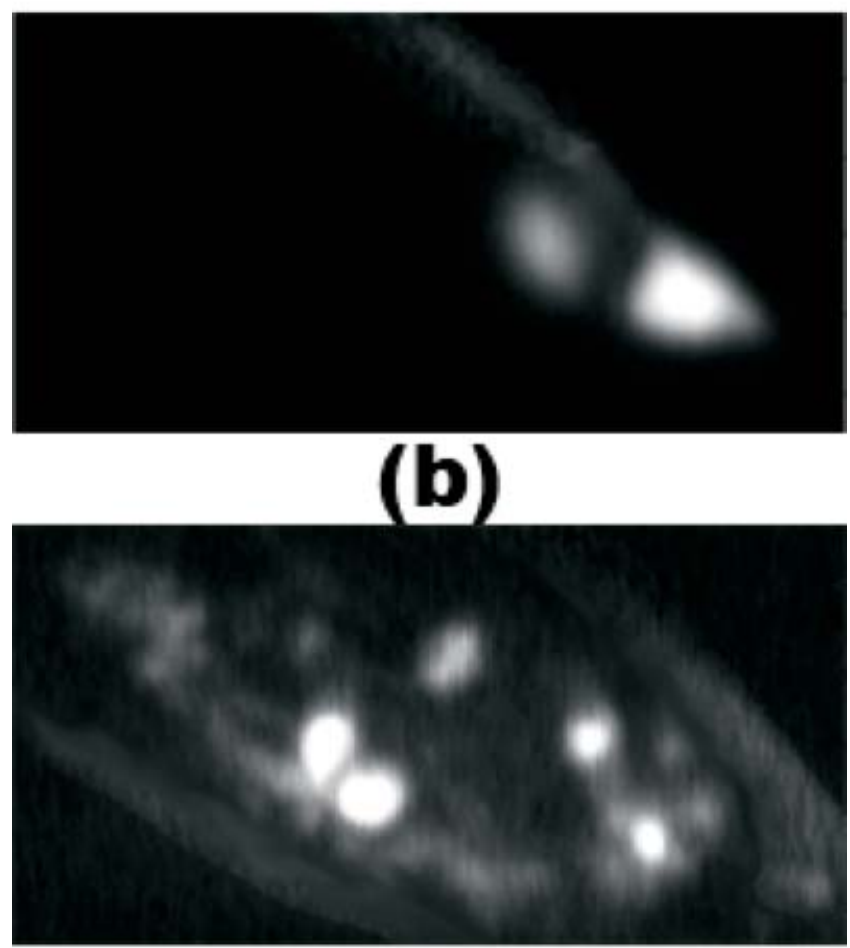

(c)

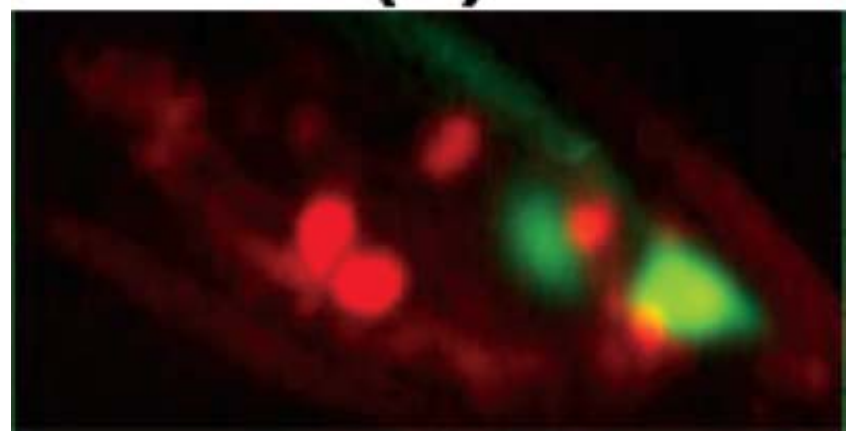

Fig. 7. Non-linear signals generated at the tail of an animal expressing GFP in the mechanoreceptor neurons: (a) TPEF, (b) THG and (c) multi-modal image obtained by the combination of the previous two: TPEF (green), THG (red). The spatial resolution is improved by replacing the xy motorized stage with galvano-mirrors.

system (6220H Cambridge Technology Inc.). Figure 7 shows the non-linear images (7(a) TPEF, 7(b) THG and 7(c) the combination of the two previous contributions; TPEF in green, THG in red), obtained from the tail of an animal expressing GFP in the mechanoreceptor neurons. The scanning area is $30 \times 15 \mu^{2}$ with 200 and 100 points resolution, respectively ( 1 pixel $\sim 150 \mathrm{~nm}$ ). The precise localization of the two touch receptor neurons and one neuron axon is feasible via TPEF measurements. Consequently, by employing 
this configuration, new anatomical features such as the axon morphology of worm neurons $(\sim 200 \mathrm{~nm})$ are more easily detectable. We note that, for the THG measurements (Fig. 7(b)), the main signal contributors (discontinuities in the tail of the animal) are similar with the ones detected by employing the xyz Standa system (Figs 2(b) and 4(c)).

\section{Conclusions}

The application of novel imaging techniques, such as nonlinear microscopy and non-linear ionization, in vivo, towards the elucidation of fundamental biological mechanisms is drawing the attention of an increasing number of researchers. These imaging technologies represent the forefront of research in cell biology. In this study, we demonstrate the potential of third-harmonic generation (THG) measurements to image specific cellular and anatomical features of C. elegans. We find that THG is a powerful tool for probing structural and anatomical changes of biological samples and processes at the microscopic level. This is the first time that a THG modality is used to image $C$. elegans animals. To this end we have developed a compact, reliable, inexpensive non-linear imaging system. Femtosecond laser pulses $(1028 \mathrm{~nm})$ were utilized for the excitation of biological samples. The emitted THG signal lies in the near-UV part of the spectrum $(343 \mathrm{~nm})$. Consummative and specific information about the anatomy of the nematode was collected by implementing a combination of THG, SHG and TPEF image contrast modalities on the same microscope. In addition, 3D reconstruction of TPEF and THG images was performed in order to achieve the 3D delineation of the outline, organs (pharynx) and specific cell types (neurons) of the worm. Moreover, a number of experiments were performed by employing a galvano-mirror scanning system in order to improve the quality and resolution of the recorded non-linear images.

We anticipate that the development and availability of an imaging station combining THG, SHG and TPEF will facilitate anatomical and functional studies at microscopic level in many biological settings. In C. elegans, the ability to perform simultaneous THG, SHG and TPEF imaging allows coupling of structural information extraction with monitoring of specific cellular physiological parameters, such as membrane potential in neurons (Khatchatouriants et al., 2000). Given the wealth of information on the biology of this organism and the broad range of genetic and molecular techniques applicable, this system will aid in-depth investigations of how genes specify and control neuron function to generate behaviour. Finally, while in this study we used C. elegans to demonstrate the imaging capacity of our experimental set-up, it can be readily adapted for work with diverse organisms and a variety of tissues.

\section{Acknowledgements}

This work was supported by the UV Laser Facility operating at IESL-FORTH under the European Commission program
'Improving Human Research Potential' (RII3-CT-2003506350) and by the Marie Curie Transfer of Knowledge project 'NOLIMBA' (MTKD-CT-2005-029194).

\section{References}

Bloembergen, N. (1965) Nonlinear optics. 4th Ed. World Scientific, Singapore.

Brenner, S. (1974) The genetics of Caenorhabditis elegans. Genetics 77, 7194.

Campagnola, P.J., Clark, H.A., Mohler, W.A., Lewis, A. \& Loew, L.M. (2001) Second-harmonic imaging microscopy of living cells. J. Biomed. Opt. 6 , 277-286.

Campagnola, P.J., Millard, A.C., Terasaki, M., Hoppe, P.E., Malone, C.J. \& Mohler, W.A. (2002) Three-dimensional high-resolution secondharmonic generation imaging of endogenous structural proteins in biological tissues. Biophys. J. 81, 493-508.

Campagnola, P.J. \& Loew, L.M. (2003) Second-harmonic imaging microscopy for visualizing biomolecular arrays in cells, tissues and organisms. Nat. Biotechnol. 21, 1356-1360.

Chu, S.W., Chen, I.H., Liu, T.M., Chen, P.C., Sun, C.K. \& Lin, B.L. (2001) Multimodal nonlinear spectral microscopy based on a femtosecond Cr:forsterite laser. Opt. Lett. 26, 1909-1911.

Chu, S.W., Chen, I.H., Liu, T.M., et al. (2002) Nonlinear bio-photonic crystal effects revealed with multimodal nonlinear microscopy. J. Microsc. 208, 190-200.

Chu, S.W., Chen, S.Y., Tsai, T.H., Liu, T.M., Lin, C.Y., Tsai, H.J. \& Sun, C.K. (2003) In vivo developmental biology study using noninvasive multiharmonic generation microscopy. Opt. Express 11, 3093-3099.

Clay, G.O., Millard, A.C., Schaffer, C.B., Aus-der-Au, J., Tsai, P.S., Squier, J.A. \& Kleinfeld, D. (2006) Spectroscopy of third-harmonic generation: evidence for resonances in model compounds and ligated hemoglobin. J. Opt. Soc. Am. B 23, 932-950.

Curcio, J.A. \& Petty, C.C. (1951) The near infrared absorption spectrum of liquid water. J. Opt. Soc. Am. 41, 302-304.

Debarre, D., Supatto, W. \& Beaurepaire, E. (2005) Structure sensitivity in third-harmonic generation microscopy. Opt. Lett. 30, 21342136.

Debarre, D., Supatto, W., Pena, A.M., et al. (2006) Imaging lipid bodies in cells and tissues using third-harmonic generation microscopy. Nat. Methods 3, 47-53.

Denk, W., Strickler, J.H. \& Webb, W.W. (1990) Two-photon laser scanning fluorescence microscopy. Science 248, 73.

Dombeck, D.A., Blanchard-Desce, M. \& Webb, W.W. (2004) Optical recording of action potentials with second-harmonic generation microscopy. J. Neurosci. 24, 999-1003.

Filippidis, G., Kouloumentas, C., Voglis, G., Zacharopoulou, F., Papazoglou, T.G. \& Tavernarakis, N. (2005a) Imaging of Caenorhabditis elegans neurons by second harmonic generation and two-photon excitation fluorescence. J. Biom. Opt. 10, 024015.

Filippidis, G., Kouloumentas, C., Kapsokalyvas, D., Voglis, G., Tavernarakis, N.\& Papazoglou, T.G. (2005b) Imaging of Caenorhabditis elegans samples and sub-cellular localization of new generation photosensitizers for photodynamic therapy, using non-linear microscopy. J. Phys. D: Appl. Phys. 38, 2625-2632.

Khatchatouriants, A., Lewis, A., Rothman, Z., Loew, L. \& Treinin, M. (2000) GFP is a selective non-linear optical sensor of electrophysiological processes in Caenorhabditis elegans. Biophys. J. 79, 23452352. 
Lewis, A., Khatchatouriants, A., Treinin, M., et al. (1999) Second harmonic generation of biological interfaces: probing the membrane protein bacteriorhodopsin and imaging membrane potential around GFP molecules at specific sites in neuronal cells of C. elegans. Chem. Phys. 245, 133-144.

Mello, C.C., Kramer, J.M., Stinchcomb, D. \& Ambros, V. (1991) Efficient gene transfer in C. elegans: extrachromosomal maintenance and integration of transforming sequences. EMBO J. 10, 3959-3970.

Millard, A.C., Wiseman, P.W., Fittinghoff, D.N., Wilson, K.R., Squier, J.A. \& Muller, M. (1999) Third-harmonic generation microscopy by use of a compact, femtosecond fiber laser source. App. Opt. 38, 7393-7397.

Millard, A.C., Jin, L., Lewis, A. \& Loew, L.M. (2003) Direct measurement of the voltage sensitivity of second-harmonic generation from a membrane dye in patch-clamped cells. Opt. Lett. 28, 1221-1223.

Mohler, W., Millard, A.C. \& Campagnola, P.J. (2003) Second harmonic generation imaging of endogenous structural proteins. Methods 29, 97 109.

Moreaux, L., Sandre, O., Blanchard-Desce, M. \& Mertz, J. (2000) Membrane imaging by simultaneous second-harmonic and two-photon microscopy. Opt. Lett. 25, 320-322.

Moreaux, L., Pons, T., Dambrin, V., Blanchard-Desce, M. \& Mertz, J. (2003) Electro-optic response of second-harmonic generation membrane potential sensors. Opt. Lett. 28, 625-627.

Muller, M., Squier, J., Wilson, K.R.\& Brakenhoff, G.J..(1998) 3D microscopy of transparent objects using third-harmonic generation. J. Microsc. 191, 266-274.

Nikolenko, V., Nemet, B. \& Yuste, R. (2003) A two photon and second harmonic microscope. Methods 30, 3-15.

Plotnikov, S.V., Millard, A.C., Campagnola, P.J. \& Mohler, W.A. (2006) Characterization of the myosin-based source for second-harmonic generation from muscle sarcomeres. Biophys. J. 90, 693-703.

Pons, T., Moreaux, L., Mongin, O., Blanchard-Desce, M. \& Mertz, J. (2003) Mechanisms of membrane potential sensing with second-harmonic generation microscopy. J. Biomed. Opt. 8, 428-431.

Squier, J. \& Muller, M. (2001) High resolution nonlinear microscopy: A review of sources and methods for achieving optimal imaging. Rev. Sci. Instrum. 72, 2855-2867.

Squier, J.A., Muller, M., Brakenhoff, G.J. \& Wilson, K.R. (1998) Third harmonic generation microscopy. Opt. Express 3, 315-324.

Sulston, J.E., Schierenberg, E., White, J.G. \& Thomson, J.N. (1983) The embryonic cell lineage of the nematode Caenorhabditis elegans. Dev. Biol. 100, 64-119.

Sun, C.K., Chu, S.W., Chen, S.Y., Tsai, T.H., Liu, T.M., Lin, C.Y. \& Tsai, H.J. (2004) Higher harmonic generation microscopy for developmental biology. J. Struct. Biol. 147, 19-30.

Supatto, W., Debarre, D., Fargea, E. \& Beaurepaire, E. (2005) Femtosecond pulse-induced microprocessing of live Drosophila embryos. Med. Las. Appl. 20, 207-216.

The C. elegans Sequencing Consortium (1998) Genome sequence of the nematode C. elegans: a platform for investigating biology. Science 282, 2012-2018.

Tsai, T.H., Tai, S.P., Lee, W.J., Huang, H.Y., Liao, Y.H. \& Sun, C.K. (2006) Optical signal degradation study in fixed human skin using confocal microscopy and higher-harmonic optical microscopy. Opt. Express 14, 749-758.

White, J.G., Southgate, E., Thomson, J.N. \& Brenner, S. (1996) The structure of the nervous system of Caenorhabditis elegans. R. Soc. London B: Biol. Sci. 314, 1-340.

Williams, R.M., Zipfel, W.R. \& Webb, W.W. (2005) Interpreting secondharmonic generation images of collagen I fibrils. Biophys. J. 88, 13771386.

Xu, C., Zipfel, W., Shear, J.B., Williams, R.M. \& Webb, W.W. (1996) Multiphoton fluorescence excitation: new spectral windows for biological nonlinear microscopy. Proc. Natl. Acad. Sci. U.S.A. 93, 1076310768.

Yeh, T., Nassif, N., Zoumi, A. \& Tromberg, B.J. (2002) Selective corneal imaging combined second-harmonic generation and twophoton excited fluorescence. Opt. Lett. 27, 2082-2084.

Yelin, D., Oron, D., Korkotian, E., Segal, M. \& Silberberg, Y. (2002) Thirdharmonic microscopy with a titanium-sapphire laser. Appl. Phys. B 74, 97-101.

Yelin, D. \& Silberberg, Y. (1999) Laser scanning third-harmonicgeneration microscopy in biology. Opt. Express 5, 169-175.

Zipfel, W.R., Williams, R.M., Christie, R., Nikitin, A.Y., Hyman, B.T. \& Webb, W.W. (2003) Live tissue intrinsic emission microscopy using multiphoton-excited native fluorescence and second harmonic generation. Proc. Natl. Acad. Sci. U.S.A. 100, 7075-7080.

Zoumi, A., Yeh, A. \& Tromberg, B.J. (2002) Imaging cells and extracellular matrix in vivo by using second-harmonic generation and two-photon excited fluorescence. Proc. Natl. Acad. Sci. U.S.A. 99, 11014-11019. 